\title{
Band Structure and Spectroscopy of Graphene-Pd Hybrids
}

\author{
Micah Eastman ${ }^{1}$, Wen Qian ${ }^{1}$, Daniel Hedinger ${ }^{1}$, Alex Jacobson ${ }^{1}$, Bin Jiang ${ }^{2}$ and Jun Jiao ${ }^{1}$ \\ 1. Portland State University, Department of Physics, Portland OR, USA \\ 2. Portland State University, Department of Mathematics and Statistics, Portland OR, USA
}

To evaluate the electronic and structural implications of graphene-Pd composite materials, samples of graphene were prepared and hybridized with $\mathrm{Pd}$ nanoparticles in accordance with our previously reported thermal-expansion, liquid-exfoliation, solvothermal-reaction (TELESR) process [1]. The resulting graphene-Pd hybrid was evaluated by bright-field (BF) and high-resolution (HR) transmission electron microscopy (TEM), as well as selected-area electron diffraction (SAED) in a FEI Tecnai F20 TEM operated at $80 \mathrm{keV}$. Analysis (Fig. 1) shows that the Pd particles are approximately $40 \mathrm{~nm}$ in diameter and are well distributed on the graphene surface, that the graphene lattice remained relatively high quality through the hybridization process, and that the expected FCC-Pd polycrystalline reflections were observed. Further structural and solid state properties were probed by Raman and electron energy loss (EEL) spectroscopy (Fig. 2). These results were compared to band structure calculations (Fig. 3).

Raman spectra were collected with a $532 \mathrm{~nm}$ source using a HORIBA LabRAM800 spectrometer. Two principle observations were made: (1) that the graphene sheets were predominantly 4-5 layers thick, as indicated by the lineshape of the 2D-band, and (2) that the particle deposition process was likely dominant at defect sites in the graphene lattice. The 2D- and D-bands are both created by doubleresonance processes involving iTO phonons - the 2D-band is caused by $\pi$-to- $\pi^{*}$ excited electron scattering inelastically off of two iTO phonons whereas the D-band is caused by inelastic scattering off of one iTO phonon and elastic scattering off of a defect site [2]. After hybridization, relative to the Gband, the integrated intensity of the 2D-band increased by $13.5 \%$ whereas the D-band decreased by $36.6 \%$, which suggests a reduction in defect scattering events.

Background-subtracted EEL spectra are shown in Figure 2b. The spectra were normalized by equating the $\pi$-plasmon peak intensities to highlight other spectral features (graphene-Pd exhibited a slightly reduced peak intensity, though still within $95 \%$ of the unhybridized graphene). Several features are of note: (1) a slight broadening of the $\pi$-plasmon peak and a minor red-shifting of $0.19 \mathrm{eV}$ (from 6.59 to $6.40 \mathrm{eV}$ ), (2) a similarly red-shifted (from 27.3 to $26.9 \mathrm{eV}$ ) and broadened $\pi+\sigma$ plasmon peak, and (3) a less distinct minima between the $\pi$ and $\pi+\sigma$ plasmon peaks. These differences may be attributed to changes in the electronic band structure, which were computed for 4-layer graphene and graphene-Pd systems using the VASP software package (Fig. 3). A slight narrowing of the $\pi-\pi^{*}$ band gap is observed near the M-point, and these bands exhibit an increased dispersion (exact values are presented in Figure $3 \mathrm{c}$ ), which may account for the broadening and shift observed in the $\pi$ plasmon peak. Additionally, transitions to the $\sigma^{*}$ bands which contribute towards the $\pi+\sigma$ plasmon near $27 \mathrm{eV}$ [3] were likely redshifted due to the deeper-lying $\sigma^{*}$ bands near the $\Gamma$ and $\mathrm{M}$ points in the hybridized structure. This may be responsible for the remaining observations in the EEL spectra.

\section{References:}

[1] W. Qian et al, Green Chemistry 14 (2012), p. 371

[2] L.M. Malard et al, Physics Reports 473 (2009), p. 51 
[3] A. Marinopoulos et al, Physical Review B 69 (2004), p. 245419

[4] This work was supported by the NSF under award \#1057565 and REU site \#1004737
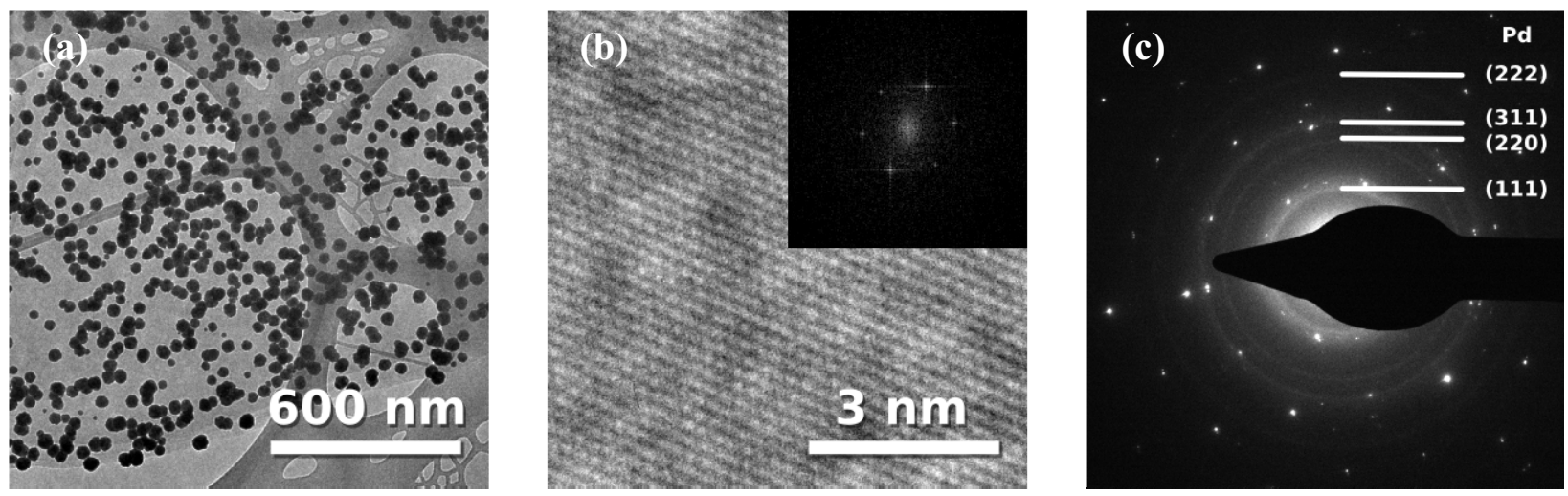

Figure 1. (a) BF-TEM, (b) HRTEM and inset FFT diffractogram, and (c) SAED pattern.
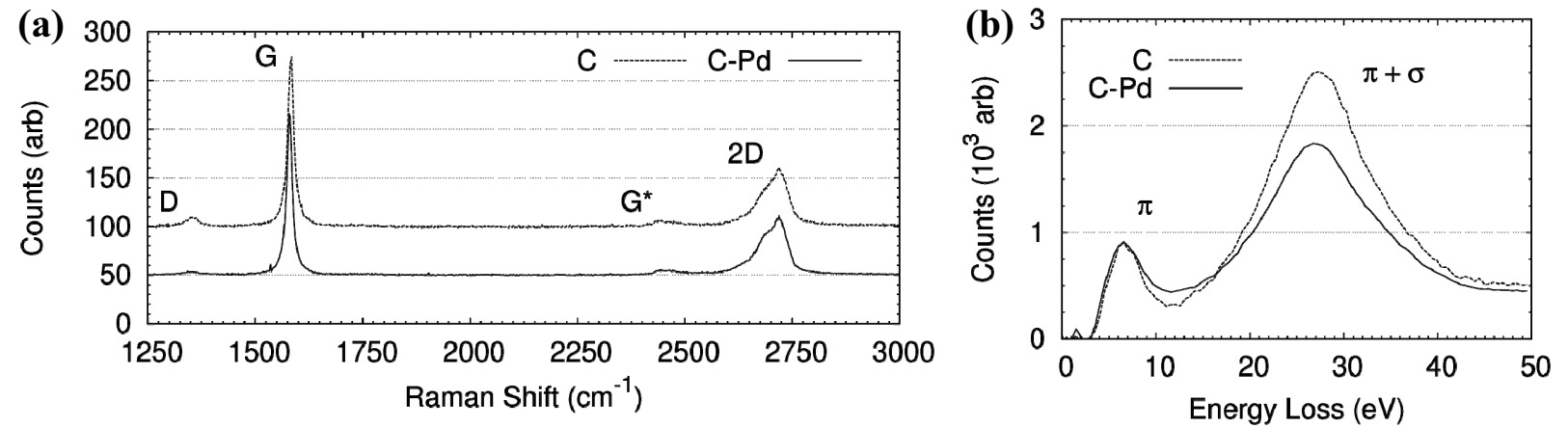

Figure 2. (a) Raman and (b) EEL spectra of graphene and graphene-Pd hybrid.
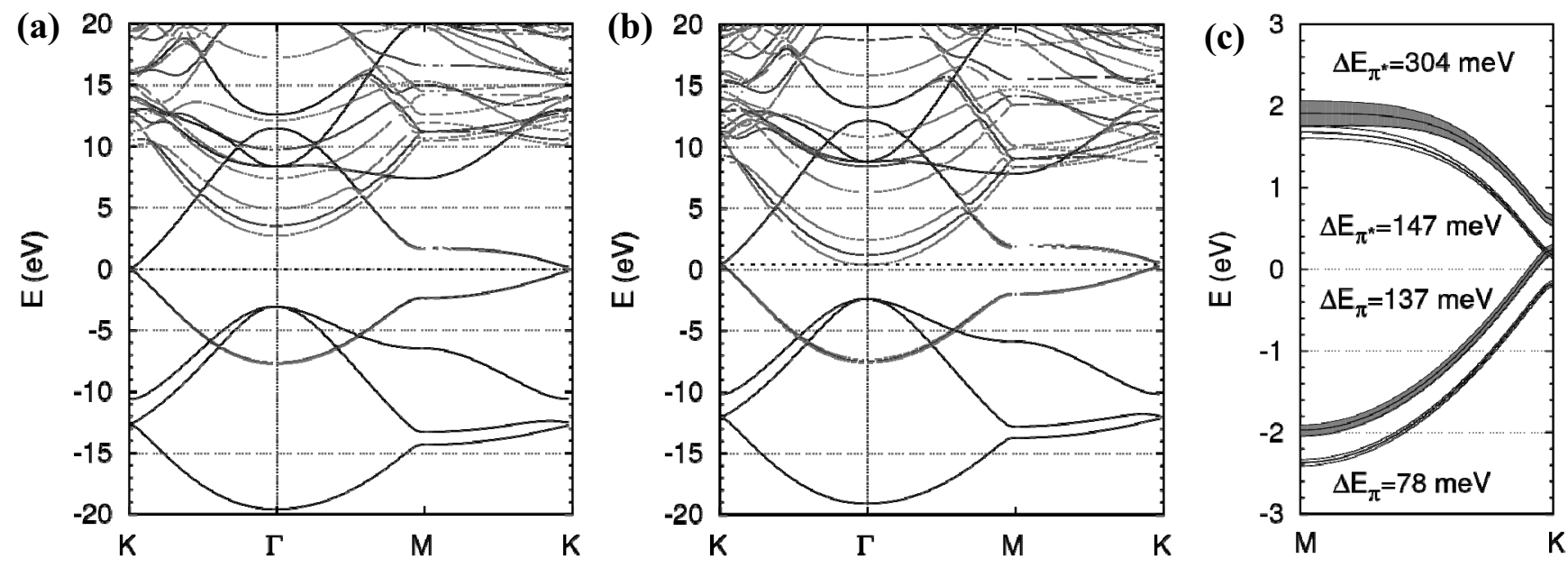

Figure 3. Band structures of (a) 4-layer graphene and (b) 4-layer graphene hybrid. (c) KM region of both band structures with enlarged $\pi$ and $\pi^{*}$ bands (shaded region denotes the graphene-Pd bands). 